\title{
Analyzing the Viability of UAV Missions Facing Cyber Attacks
}

\author{
Jukka Soikkeli \\ Department of Computing \\ Imperial College London \\ London, UK \\ j.soikkeli@imperial.ac.uk
}

\author{
Cora Perner \\ Airbus Cybersecurity $\mathrm{GmbH}$ \\ Taufkirchen, Germany \\ cora-lisa.perner@airbus.com
}

\author{
Emil C. Lupu \\ Department of Computing \\ Imperial College London \\ London, UK \\ e.c.lupu@imperial.ac.uk
}

\begin{abstract}
With advanced video and sensing capabilities, unoccupied aerial vehicles (UAVs) are increasingly being used for numerous applications that involve the collaboration and autonomous operation of teams of UAVs. Yet such vehicles can be affected by cyber attacks, impacting the viability of their missions. We propose a method to conduct mission viability analysis under cyber attacks for missions that employ a team of several UAVs that share a communication network. We apply our method to a case study of a survey mission in a wildfire firefighting scenario. Within this context, we show how our method can help quantify the expected mission performance impact from an attack and determine if the mission can remain viable under various attack situations. Our method can be used both in the planning of the mission and for decision making during mission operation. Our approach to modeling attack progression and impact analysis with Petri nets is also more broadly applicable to other settings involving multiple resources that can be used interchangeably towards the same objective.
\end{abstract}

Index Terms-UAV, cyber attack, mission viability analysis, Petri net, cyber resilience, cyber security

\section{Introduction}

Among the various uses for unoccupied aerial vehicles $(\mathrm{UAVs})^{1}$, several applications involve the operation and collaboration of teams of multiple UAVs, for example search and rescue, delivery of goods, providing wireless coverage, and fire survey [1]. In a firefighting context, the use of UAVs enables effective survey of the area whilst reducing human exposure to danger. UAVs are currently used for this purpose in, for example, Latvia and the Netherlands [2]. However, UAVs are also vulnerable to various cyber attacks, including ones that enable UAV hijacking and allow the attacker to gain complete control of the vehicle. Several works, such as [3]-[5], analyze UAV security and survey the different attacks demonstrated so far on UAVs. As the capabilities of UAVs increase, so

NB: appendices, if any, did not benefit from peer review.

This work has been partially supported by the European Union's Horizon 2020 Research and Innovation program under the CONCORDIA project grant agreement No 830927. Jukka Soikkeli was supported by the EPSRC Centre for Doctoral Training in High Performance Embedded and Distributed Systems (HiPEDS, Grant Reference EP/L016796/1).

1. In this work we use the term UAV to refer to the vehicle, regardless of whether it is autonomous or remotely controlled by a human pilot. does their attack surface [5], and new types of attacks can be anticipated.

For the successful running of missions with multiple UAVs, it is important to be able to assess the impact of a cyber attack on mission performance, to determine mission viability after attack and to enable planning missions with appropriate levels of redundancy. This requires methodologies that can simultaneously analyze both the propagation of the attack and its impact on the overall mission, taking into account mission requirements and component inter-dependencies. To this end, we propose a methodology for modeling and reasoning about attacks and mission impacts via the combined use of Petri nets and a model of mission performance. We demonstrate our approach through a case study of fire survey missions.

Our approach is a methodology for performance-based analysis of resilience to cyber attacks with a focus on mission viability, and as such it is intended to have wide applicability. It uses a Petri net model to estimate the impact of an attack that can spread within the team of UAVs given the system configuration, defensive capabilities in terms of containment and recovery, and the expected attack behaviors. Petri nets are a framework for modeling distributed systems, especially useful for modeling the behavior of systems with multiple components that exhibit symmetry in how they function as part of the system.

Our evaluation of attack impacts relates to works on attack impact analysis such as [6], [7], but differs in the use of Petri nets for the attack modeling, and in the application to UAVs. While Petri nets have been used for modeling cyber attacks by e.g. [8]-[11], our approach differs from them in employing the Petri net model as part of a performance impact evaluation model, in the attack modeling specifics, and the application to UAV missions.

Our key contributions are: a) We propose a novel methodology for modeling and reasoning about attack impacts for UAV missions. Our attack propagation model is particularly suitable for modeling attacks that can spread across a team of UAVs, as the Petri net formalism enables a compact and effective modeling of impacts to systems with multiple interchangeable components which can be attacked in a similar manner. b) We demonstrate how this methodology can be used both during mission planning and as decision support tool during mission operation. The approach helps to quantify things such as: i) The expected mission performance if an attack occurs at different times during the mission, given the mission configuration and redundancy; ii) The number of redundant UAVs required 
for a desired likelihood of success; iii) How the number of vehicles required changes with the speed of containment relative to attack speed. c) We formalize and analyze in detail a case study of wildfire surveys, explaining the use and benefits of the methodology.

The paper is structured as follows: Section 2 discusses related work. Our case study and threat model are introduced in Section 3. Our methodology for mission viability analysis is described in Section 4, and its implementation in our case study is shown in Section 5. Section 6 discusses our analysis results, and Section 7 concludes.

\section{Related work}

UAV security from the point of view of individual vehicles has been studied by e.g. [3]-[5]. Additionally, Javaid et al. [12] proposed an approach to model and analyze the treats to a system of several UAVs. While their approach is a framework for estimating risk from various specific attacks on a system, ours is a high level model intended for estimating mission viability in response to attacks that impact the availability of vehicles, with a focus on the performance of the overall mission.

We evaluate the impacts of cyber attacks on system performance over time and on mission viability. In this regard, our approach relates to works on quantifying cyber resilience [13] of a system, most directly to approaches for measuring resilience based on performance over time such as Bruneau et al. [14] and Ganin et al. [15]. In employing a resilience-inspired view on attack impacts as a basis for decision making, our approach is similar to that adopted in [7] to select countermeasures to attacks, but our focus here is on UAVs and on making decisions over the appropriate mission setup and on mission continuation in the face of attacks. We model attack impacts with a combination of a model of attack progression and a model of system performance in producing its output. This relates to methods by [6], [7] that approach attack impact assessment via a combination of an attack graph (AG) and a service dependency graph. In contrast to these works, we use a Petri net for modeling attack progression, and find it particularly suited to this in our application context. While the use of AGs for describing attack progression works well when the attacker's goal is reaching a specific privilege, cases where the attacker aims to reduce system performance and availability by spreading across components are more accurately and more conveniently modeled using Petri nets. Similarly, our application area contains multiple interchangeable components that share the same vulnerabilities, which is more difficult to represent using AGs, but suitable for Petri nets.

Petri net formalisms have been used before for attack modeling in works such as [8]-[11]. Madan and Trivedi [8] proposed using generalized stochastic Petri nets (GSPNs) as a tool to solve a security quantification model that incorporates attacks and responses, an attack graph variant called attack response graph (ARG). Their approach is to closely replicate the ARG in GSPN form. The method proposes a straightforward conversion of standard AGs to GSPNs; however, it yields a complex representation using a high number of places and transitions as each AG node is given its own place and related transitions. In complex missions containing multiple functionally equivalent components, our method for describing attacks leverages these symmetries to generate more compact models that are easier to analyze and use.

Li et al. [9] use a GSPN to model the impact of coordinated topology attacks to smart grids. Their use of a GSPN for attack impact modeling in the presence of countermeasures is similar to our evaluation of expected attack impacts. However, a key difference in the attack modeling is how the attack progresses: the propagation of the compromise from a UAV to another is a key feature of our model, while they model attacks to different components as individual attacks without a direct link between compromises.

Some works have extended features that add to the expressiveness of the Petri net formalism, at the expense of the closed-form solution method. For example, Dahl and Wolthusen [10] introduced a Petri net formalism for attack scenarios where the transition firing times are distributed within an interval, while Collins and Huzurbazar [11] propose a formalism that allows for arbitrary firing time distributions. Such models require simulated solution, as they do not satisfy the memoryless property that would allow them to be solved as a CTMC. Simulation approaches have benefits such as supporting added model features, but drawbacks on solution time due to the numerous iterations required to obtain approximate average behavior. However, as the simulation tools are not shared by the authors and the analyses and models are used in different contexts, direct comparisons are not possible.

\section{Scenario and Threat Model}

Our case study is a survey mission involving a team of UAVs in a wildfire scenario. To give context on the scale of the issue of wildfires, in European countries on average 444000 ha were burnt by forest fires annually over the last decade (2010-2019), according to data from the European Forest Fire Information System (EFFIS) [16]. For an example of the economic loss incurred, the direct economic damage from forest fires in Germany in 2019 was estimated to be $€ 819$ per hectare burnt [2].

Our case study focuses on wildfires that are large enough to benefit from multiple UAVs to map the potential fire area. Depending on the equipment used and the speed achievable, this will likely mean fires where the required area coverage is over a hundred hectares. For reference, according to data from [2], within Europe there were over a hundred fires exceeding 100 ha burnt area in 2019, with the largest single fires covering thousands of hectares.

Our scenario has the following features: The mission contains a team of surveying vehicles (UAVs) flying in a loose formation across a given area to identify wildfires by collecting data. The data are passed to a centralized mission control (MC), which deals with processing the data and coordinating the overall mission. The data collected by the UAVs contains camera feed of the area (UAVs with video sensors), and temperature of fires (temperature sensors), and maps based on these. A subset of the UAVs are directly connected to the mission control via radio/line of sight communication, while communication between the UAVs within a team is ensured by some of the UAVs acting in a relay role. The data thus flows to the MC via 
the inter-vehicular network across relays, and via vehicles with a direct link to the MC.

Threat model: In our model we consider attacks that disable the vehicles' sensing capabilities, via "manual" or automated attacks, including malware. The key difference between the attack types is the speed at which the attack spreads to other vehicles. We do not model the modes of attack in detail, but reflect different attack speeds via varying parameter values.

As the focus of our work is on the overall mission viability, we do not detail how the attack proceeds within an individual UAV. That is, we assume that the attack actions within a given UAV will either succeed and compromise the UAV (and possibly propagate to other UAVs), or the attack to that UAV will fail.

We make the following assumptions on attacks: The attacker's goal is to disrupt the mission by disabling UAV capabilities or the UAVs themselves, using methods such as malware, vulnerability exploits (disabling the UAV or a sensor). Any UAV can serve as the entry node for the attack. We consider attacks that can propagate from vehicle to vehicle via lateral movement enabled by privilege escalation, i.e. a compromised UAV can be used to compromise other UAVs in the network. The impact of a compromise is the capabilities of the UAV becoming fully disabled, although the model could be extended to represent partial degradation of function for the UAVs by considering the roles they can fulfill in the mission. We consider the capabilities independently from the vehicle, which could enable cases where a UAV with one compromised capability could be used in another role that it still had a capability for, if the compromise was isolated from affecting it. In this paper we simplify by not allowing compromised UAVs to remain in the mission, but the model could be extended to consider scenarios where they could be reassigned to other roles.

\section{Methodology}

We now explain our overall methodology for performance-based resilience analysis, and then set out how it is applied to the fire survey mission. The key components of our method are:

- An attack model, describing the progression of attacks among the participant UAVs, and how they can impact parts of the production model.

- A production model, describing how the UAVs combine their functions to generate progress toward the mission objective. This is used for relating the individual components to the success of the mission.

- A definition of system performance relative to the mission objective, based on the production model. This is required for measuring attack impact.

- A model for choosing actions based on the system performance, expectations of mission progress and the costs of the actions.

The different components require input from the mission plan and the UAV connectivity status. The production model is based on the mission plan, and the specifications and capabilities of the UAVs taking part in the mission. The connectivity among the participant UAVs impacts both the attack progression and the production model, as connectivity is required to transmit the mission data.
This modular structure highlights the key parts of the methodology, and allows each component to be kept simple. This is intended to make the overall method easier to understand at a high level, simple to extend within the different modules, and easily applicable in different settings even if one of the components needs change. For example, although a performance model could be built as a Petri net and the attack and recovery dynamics could be built into the same network, such an approach would lead to more complex Petri net models, and ones that are specific to the system in question. In contrast, our method has generic aspects that make it simple to adapt to different scenarios via changes to parameters and edits to the model components, such as changing the form of the production function used to estimate system performance.

Within our fire survey scenario, the objective is to track mission progress, making decisions on whether the mission can no longer succeed and should be aborted, given the impacts of cyber attacks. In this setting, the components become the following: The production model describes how the tasks (UAV roles) combine to produce the mission output (e.g. coverage of a certain area). We model this with a Leontief production function ${ }^{2}$, where a unit of coverage rate is achieved by a strict ratio of UAVs in specific roles. We choose this form as relay roles are typically not substitutes for sensor roles and vice versa, although in a different setting a more general type of function could be used. The attack model focuses on the availability of UAV capabilities, given the threats of malware or attacks to sensors. The model describes the progression of the attacker (behavior and time to progress) within the team of UAVs. To measure performance, we set a target for area coverage given mission goals, and measure performance as the rate of the area covered per a unit of time, estimated based on the UAV type information and assuming effective control of flight paths. The decisions are to continue mission if current and expected progress meet the target, otherwise decide between using redundancy (with existing redundant capacity, or by adding UAVs) or aborting the mission.

The choice of actions is made based on current progress and expectations over what could happen during the time remaining before mission end. The decision process takes inputs from the various model components, and leads to changes in the states of the models when actions are taken. For example, adding a UAV will add capacity to the production model, but also more paths for an attack to progress. These changes can affect the performance estimates and expected mission progress. We consider the key decision to be over continuing the mission or aborting: Continue without changes if the mission can be completed without adaptations; Take a recovery action (replace a UAV with another) to improve coverage rate if recovery allows successful mission completion; Abort mission if it cannot be completed successfully even with recovery actions, or if no recovery is possible.

To illustrate the decision making required, Fig. 1 shows a sample analysis for the simple case of an attack that does not spread. For this illustration, we have used the following assumptions for the mission, and the attack:

2. An introduction to different production functions, including the Leontief input-output model, can be found in e.g. [17]. 


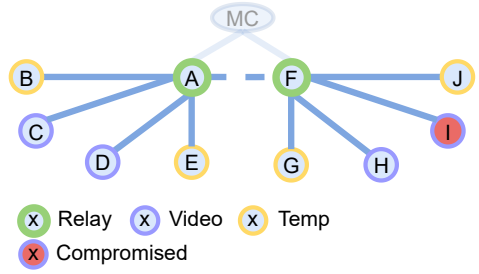

(a) Mission connectivity graph

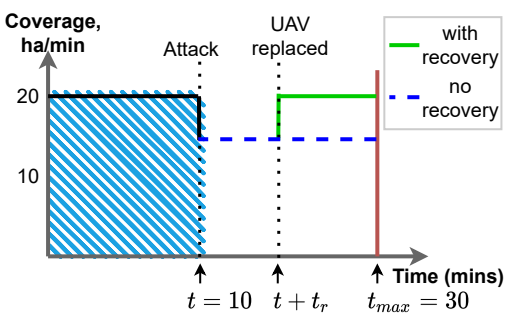

(c) Effect on mission coverage

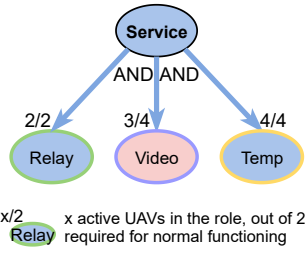

(b) Production graph

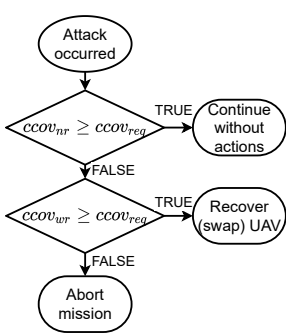

(d) Decision
Figure 1. Sample analysis, attack that does not spread

- Task: cover an area of 560 ha, within a maximum time of $t_{\max }=30$ minutes

- Coverage rate function: cov_rate $=20 *$ $\min \left(\frac{x_{r e l}}{2}, \frac{x_{v i d}}{4}, \frac{x_{t m p}}{4}\right)(h a / m i n)$, where $x_{r e l}, x_{v i d}$ and $x_{t m p}$ are the number of UAVs active in the relay, video and temperature roles, respectively. During normal operation the rate of coverage with this example function is $20 \mathrm{ha} / \mathrm{min}$.

- The attack occurs at $t=10$ (min), taking down one video sensor. As a consequence, the coverage rate drops to 15 ( $\mathrm{ha} / \mathrm{min}$ ), as $\frac{x_{v i d}}{4}=3 / 4$.

Fig. 1a shows that the attack at $t=10$ disrupts node $I$ in the mission connectivity graph, a UAV in a video capture role. The production graph in Fig. 1b shows that this impacts the video service, taking the number of UAVs in the video role below the standard requirement of 4 (shown above the "Video" node of the graph). Fig. 1c shows how this affects the mission performance in terms of coverage, with the coverage rate dropping to $15 \mathrm{ha} / \mathrm{min}$ at $t=10$. To make a decision on what to do, we must consider what could happen in the remaining time before $t_{\max }$. As the attack will not spread (in this example), there are two cases to consider. Without a recovery action, the coverage rate remains at the level of $15 \mathrm{ha} / \mathrm{min}$ until the end of the mission time. On the other hand, recovery could be done in $t_{r}$ time by replacing the compromised UAV with another UAV (without the same vulnerability), so performance would return to $20 \mathrm{ha} / \mathrm{min}$ at time $t=10+t_{r}$. Finally, Fig. 1d shows the decision to be made: a) Continue without recovery if cumulative coverage without recovery ccov $_{n r}$ meets the required level $c^{c o v} v_{r e q}$ - this is not the case here, as $\operatorname{ccov}_{n r}=500 \nsupseteq 560=$ ccov $_{r e q}$; b) Recover by swapping UAVs if coverage with recovery, $\operatorname{ccov}_{w r}$, exceeds $\operatorname{ccov}_{r e q}$ - this holds in the example if $t_{r}<8$; c) Abort the mission if $\operatorname{cov}_{w r}<\operatorname{cov}_{r e q}$ - here if $t_{r}>8$.

The example above gives a flavor of the analysis required, but in practice we expect that the attack can spread to other vehicles, or that there can be several attacks. Our attack propagation model based on Petri nets is intended for analyzing such situations. The analysis employs Petri nets as a formalism to evaluate the effects

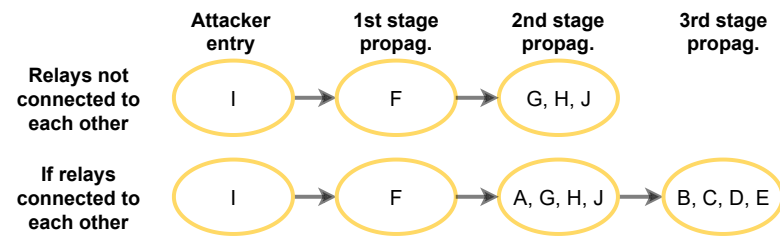

Figure 2. Potential high-level attack paths that ensue from the mission connectivity as in Fig. 1a

of an attack. This provides a stylized representation of the attack progression and defensive actions (containment and recovery), with a specific net instantiated based on the attack propagation model (showing attack paths), the attacker entry point, and assumptions on the propagation speed, defense capabilities and speed.

\section{Model implementation}

\subsection{Connectivity and attack progression}

In addition to enabling data transfer from sensors to the mission control, connectivity also leads to the existence of potential attack paths across vehicles. Fig. 2 shows how the connectivity of the surveying mission can lead to specific attack paths. In our case study we model connectivity as a hubs and spokes model, as in Fig. 1a, where all sensor UAVs are connected to relay UAVs, which then pass the information to the MC. This type of connectivity is typical of such missions. In this context, the attack progresses in a straightforward sequence of steps, with differences arising based on which node is attacked first, and whether the transmission of the attack follows a one-hop transmission or if all reachable UAVs can be infected in one step. Fig. 2 shows two alternatives for attack progression given the connectivity is as shown in Fig. 1a: 1. Relays ( $A$ and $F$ ) are not connected to each other, meaning that only half of the vehicles can become compromised by an attack on $I ; 2$. The relays are connected to each other, which makes it possible for the compromise of $F$ to also compromise $A$, and the vehicles connected to $A$. In this paper we focus on the latter case; the analysis with the separated relays is similar and yields similar insights, although it yields a smaller attack impact.

\subsection{Actions to mitigate attacks}

We consider the following remedial actions as part of possible responses to an attack:

1) Contain the attack by disabling a compromised part of the fleet (e.g. return to base). This leads to losing the UAVs in question for the duration of the mission.

2) Swap roles/tasks between UAVs. Some mission time is lost when before the swap is completed.

3) Introduce a replacement UAV to the mission. Some delay is observed before the replacement arrives.

Swapping roles acts as a recovery mechanism for performance, allowing to continue tasks at the previous coverage level, if the relevant capabilities exist in other UAVs. Introducing a new UAV into the mission achieves the same goal but takes a longer time on average, as the new UAVs need to fly in from the dispatch point. 
The effectiveness of swapping tasks between UAVs (or bringing in replacements) depends on the level of similarity between the UAVs in terms of exploitability: if it's easy to replicate the attack on the UAV swapped in, then the failure of that task is still likely. We assume that the UAV to be swapped in does not have a vulnerability to the same attack, for example as it is a UAV of a different type, although we are aware that this is a strong assumption. In future work we plan to relax this assumption and extend the model to study the impact of several successive attacks, regardless on whether they resulted from the same or different vulnerabilities.

\subsection{Petri net model}

Petri nets are especially useful for settings with multiple components which exhibit individual behavior that is symmetric across the components, but which takes place concurrently and can have interactions or joint behavior. Thus they are particularly suited to the case of teams of UAVs, as they are similar in terms of their tasks, and are assumed to respond to attacks in a similar way. However, we distinguish between the different roles (relay, sensor [video, temperature]) the UAVs have in the mission, which we model using the Petri net extension of colored tokens. We also require time to be represented in our model, which leads us to use the timed transition extension to Petri nets, specifically the version introduced in generalized stochastic Petri nets (GSPNs) [18]. Combined, these two extensions led us to opt for the stochastic well-formed net (SWN) [19] formalism for our model, as it provides both colors and stochastic transitions ${ }^{3}$.

Fig. 3 shows the SWN model of the attack propagation and defense in our fire survey scenario. It follows standard notation for GSPNs and SWNs, and was made with GreatSPN [20]. In the remainder of this section we describe the broad aspects of the model and the basic flow of events. A detailed introduction to Petri nets and the notation can be found in e.g. [18]. Table 1 lists the parameters used in the model, with brief explanations.

As shown in Fig. 3, the network is composed of three sub-models demarcated with gray frames: the Attack submodel represents the different stages of the spread of an attack within the network of vehicles; Containment models the process of containing the attack to stop further spread; Recovery contains the role swaps and vehicle replacements that can be used to recover mission performance. The sub-models interact at several transitions and places, most importantly via their impact on the count of vehicle roles that are currently fulfilled and those unfulfilled due to compromise, represented by the places $U A V s$ and Compr, respectively. Colored tokens represent vehicles in different roles: a token of color $r l$ is a UAV in the relay role, and color $r 2$ represents a sensor role. This enables tracking the number of vehicles in different roles, and the number in each role that are compromised by an attack.

An attack is represented as follows: the initial entry to the system is denoted by the Entry transition, which takes a token of color $r 1$ or $r 2$ from the places Attacker and $U A V s$, and passes a token of the same color to Compr, and

3. Technically the SWN colors can be unfolded to represent our net as a GSPN, but that is visually more complex. one colorless token each to Stagel and Containing. The subsequent spread of the attack is represented by the firing of the timed propagation transitions (Prop1,Prop2,Prop3): e.g. firing of Propl takes the token from Stagel and one from Can_prpg (and returns it back), and colored tokens from $U A V s$ representing the number of vehicles of each role that the attack can spread to in the first stage of propagation (i.e. the vehicles that the compromised UAVs can communicate with). The number of colored tokens of color $r$ taken in step $s \in\{1,2,3\}$ of an attack is given by the parameters $S_{s r}$; these parameters are set based on the number of vehicles in different roles reachable at a given stage of an attack based on the connectivity of the mission. The same number of colored tokens of each type is passed to Compr as were taken from $U A V s$, and an uncolored token is passed to the place representing the next stage of the attack, e.g. Stage 2 . For example, in Fig. 2 where entry occurs at the sensor UAV $I$, the firing of Propl takes one token of color $r l$ from $U A V s$ to represent propagation to the relay $F$, and passes one $r l$ token to Compr. Next, the firing of Prop2 takes three tokens of color $r 2$ (sensors $G, H, J$ ), and passes three $r 2$ to Compr. As in this case the relays are connected to each other, $A$ can be compromised at the second stage with Prop2, spreading to further sensor vehicles with Prop3.

While the the number of spread stages (at most three) in this sub-model is specific to our scenario, this is straightforward to extend by adding the places, transitions and arcs for any further stages needed. This model can be made to apply to different attacks and configurations of the inter-vehicular network by providing the parameters $S_{s r}$ that describe how many of vehicles in each role are impacted at each stage of the attack.

Containment is modeled as a process that can remove the possibility of the attack propagating to other vehicles. Specifically, we include a place Can_prpg representing the ability of the attack to propagate (i.e. not being contained). and containment occurs when the token in it is removed. The timed transition Contain becomes available after attacker entry as a token is passed to Containing, and when it fires a token is passed to Contained, which causes the firing of the immediate transition $t l$ that removes the token from Can_prpg, disabling the propagation transitions.

The containment action in the SWN is treated as stopping the attack from proceeding to the next stage (to the UAVs adjacent to the attack boundary), so no further vehicles are compromised by the same attack. While this representation may appear to avoid the aspect of the containment action itself leading to loss of production from the contained UAVs, the model can be interpreted in a manner where the loss of vehicles is equivalent whether they were successfully compromised or contained. That is, we view the attack stages in the SWN as representing the extent of spread which the attack could have reached in the time conditional on the previous stage being successful. With this interpretation the SWN model counts the vehicles at a given attack stage as lost, whether it is due to them being certainly compromised or being contained due to becoming untrustworthy as the attack could conceivably have reached them. This simplifies the modeling.

Recovery in the model can occur in two ways, either by replacing compromised vehicles with others not currently partaking in the mission (e.g. because they arrived 


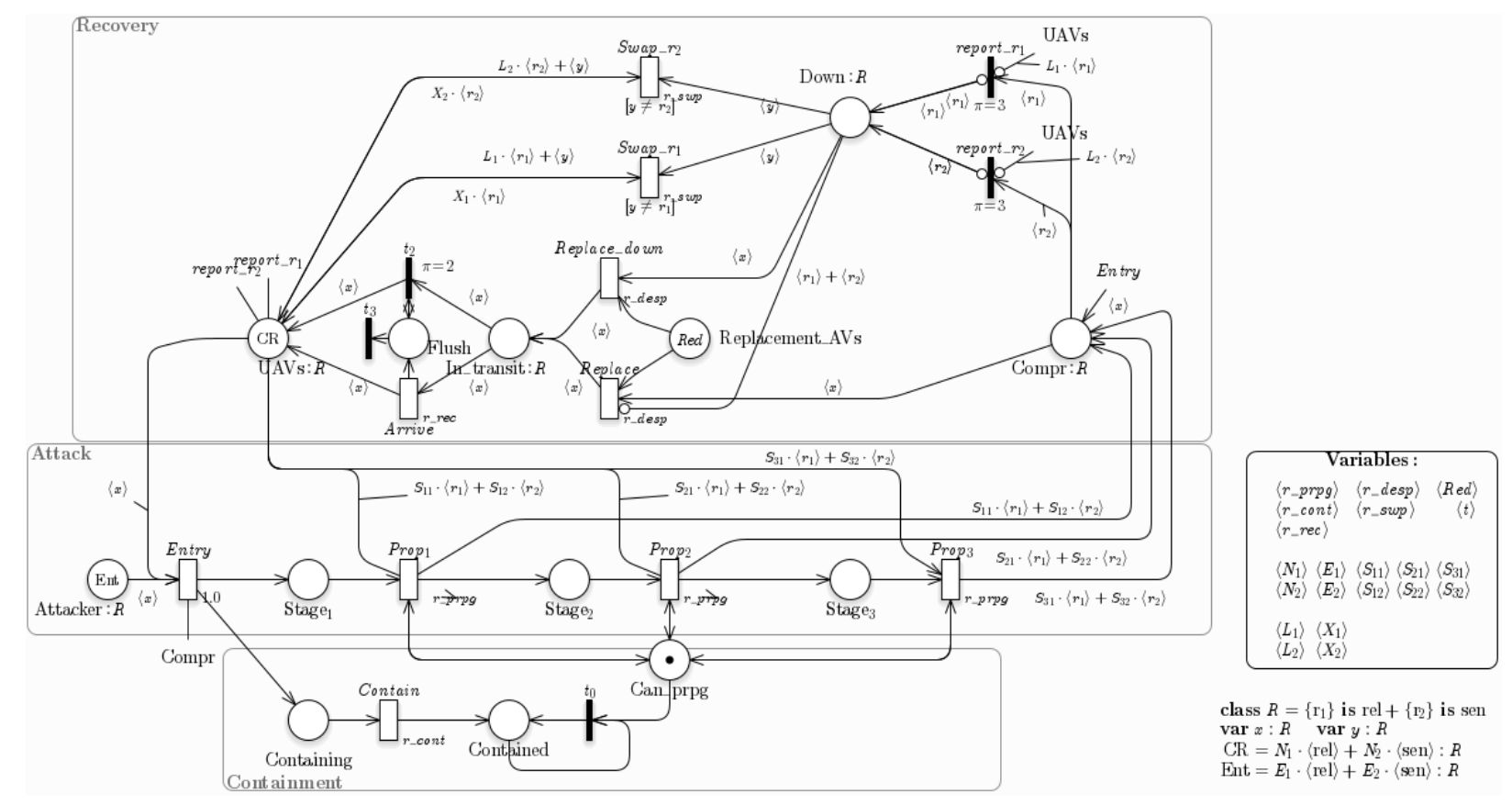

Figure 3. Stochastic well-formed net for attack stages

TABLE 1. PARAMETERS IN THE SWN MODEL

\begin{tabular}{|c|c|c|}
\hline$r_{p r p g}:$ rate of attack propagation (1/propag. delay) & $r_{\text {desp }}:$ rate of dispatch of replacements (1/desp. delay) & $r_{r e c}:$ rate of recovery via replacement (1/repl. delay) \\
\hline$r_{\text {cont }}:$ rate of attack containment ( $1 /$ cont. delay) & $r_{s w p}:$ rate of recovery via swap (1/swap delay) & $t$ : model time, seconds \\
\hline $\begin{array}{l}\text { Red: number of replacement UAVs that can be intro- } \\
\text { duced to the mission for recovery }\end{array}$ & $\begin{array}{l}N_{i} \text { : number of UAVs in role } i \text { taking part in the } \\
\text { mission }\end{array}$ & $\begin{array}{l}E_{i}: \text { number of UAVs in role } i \text { that entry occurs in, } \\
\quad E_{i} \in[0,1]\end{array}$ \\
\hline $\begin{array}{l}S_{s i}: \text { number of UAVs in role } i \text { compromised at attack } \\
\text { stage } s\end{array}$ & $\begin{array}{l}L_{i}: \text { limit UAV count in role } i \text { below which recovery } \\
\text { is necessary }\end{array}$ & $\begin{array}{l}X_{i}: \text { excess/redundancy in role } i \text { (1 over limit) } X_{i}= \\
\quad L_{i}+1\end{array}$ \\
\hline$R$ : set of vehicle roles (token colors), $R=\left\{r_{1}, r_{2}\right\}$ & $x, y$ : token color (vehicle role) variables, $x, y \in R$ & \\
\hline
\end{tabular}

to the mission after the original set of vehicles were dispatched), or by swapping the roles of vehicles already in the mission, when appropriate.

The recovery model has a multi-stage structure - when a vehicle with role $x$ has become compromised by an attack, a token of color $x$ gets added to the place Compr. If the compromise would lead to the service $x$ (e.g. the relay service) performing below the minimum requirement, a token with color $x$ is added to Down (by the firing of report_rl or report_r2), which has a higher recovery priority than tokens in Compr. If Down contains tokens, then a role swap between vehicles currently partaking in the mission can be used as a recovery mechanism to enable faster return to acceptable performance for the service.

When a token is added to Compr and no tokens are in Down, the timed transition Replace can fire, representing the step of preparing a replacement vehicle to take over the role of a compromised one. Replace cannot fire if Down has tokens, as services that are fully down take priority in recovery over those that are simply degraded. This constraint is represented by the inhibitor arc from Down to Replace. If a token exists in Down, the service flagged down can be recovered by replacing it with a new vehicle by firing Replace_down, or by swapping it with another vehicle partaking in the mission. The swap is done with either Swap_rl or Swap_r2 depending on the role to be swapped, which swaps a vehicle from a role with a redundant number of vehicles to the desired one. Once Replace or Replace_down fires, a token is placed into In_transit to represent that the replacement has been dispatched but is yet to arrive to the desired location. When the timed transition Arrive fires, the tokens that were in In_transit get passed to $U A V s$. The structure involving Flus $\bar{h}$ and immediate transitions $t 2$ and $t 3$ is used to pass all the remaining tokens from In_transit into UAVs once Arrive has fired. This is used to represent simultaneous flight of the vehicles in transit, instead of having them queue to fire "Arrive" one at a time.

This model of recovery can apply in UAV scenarios where the replacement of vehicles and swapping of roles is relevant. In a more generic setting recovery can be modeled similarly to how we model replacement, with a given recovery capacity (of components or repairers) and places and transitions representing the recovery process.

We solve the model using the GreatSPN [20] transient solver. The time unit in the model is seconds, $t \in[0,1200]$, and the transient solution is evaluated at intervals of 30 time units. The timed transitions in our model use exponentially distributed firing rates, as used by GSPN and SWN. More general distributions are enabled by some extended Petri net formalisms, but these require simulations to analyze the model.

\subsection{Mission performance modeling}

Mission performance is quantified as the rate of area covered by sensor vehicles. We approximate the expected coverage rate of an individual vehicle based on capabilities of currently available vehicles in terms of their speed and the maximum area coverage of cameras with different an- 
gles of view at plausible flight altitudes during operation. These are shown in Table 2. We have assumed speeds during operation to be between 5 and $20 \mathrm{~m} / \mathrm{s}$. For the angle of view (AOV) of UAV cameras, the reported AOV values are between $40^{\circ}$ and $95^{\circ}$ for current thermal cameras ${ }^{4}$, and normal high-definition cameras are between $80^{\circ}$ and $150^{\circ}$. We have assumed operational altitudes between 50 and $120 \mathrm{~m} .^{5}$ The resulting approximations may overestimate the coverage reachable by vehicles in practice, but provide useful direction. In practical implementations the coverage rates could be evaluated from data from previous missions.

The coverage rate of a group of UAVs on a mission is estimated by a production function, which takes into account the required number of vehicles in different roles. Here we model this according to (1):

$$
\text { cov_rate }=I C * x_{v i d}^{r e q} * \min \left(\frac{x_{r e l}}{x_{r e l}^{r e q}}, \frac{x_{s e n}}{x_{s e n}^{r e q}}\right)
$$

where $I C$ is individual coverage (as in Table 2), $x_{r l}$, is the number of UAVs active in the role $r l \in\{$ rel, sen $\}$ (relay, sensor [incl. video and temperature]), and the required number of UAVs in role $r l$ is denoted by $x_{r l}^{r e q}$. The multiplier $I C * x_{v i d}^{r e q}$ represents our assumption that the number of video sensors is a key determinant of the extent of mission coverage, while relays and temperature UAVs offer support and added services to the mission.

With the coverage rate function we can convert the SWN analysis results on UAV numbers taking part in the mission into coverage rates, evaluate mission performance and make decisions based on the expected total coverage.

The expected total coverage achievable during a mission if it is attacked is evaluated as follows: 1. The time period from the start of the mission until the attack occurs is assigned the full coverage achievable with the mission setup, e.g. if the attack occurs 5 minutes in, this is $5 *$ cov_rate. In during-mission analysis, this could instead be the actually observed coverage. 2 . The coverage from the attack moment onward is evaluated based on the results from the Petri net model, converted to coverage values using a production function such as (1). This is applied to the end of the evaluation time window of the Petri net model, unless it would exceed the overall mission time. 3. The mission time that remains after the evaluation time window of the Petri net model is assigned the coverage rate estimate reached at the end of the Petri net evaluation.

\subsection{Pre-planning and during mission use}

Our modeling provides a structured approach for mission planning support and viability evaluation. When planning for a mission, it can help determine details such as the appropriate number of vehicles to be used for a mission with a given coverage requirement, or the number of replacement vehicles for a given mission. It can also quantify the impact of various parameters on the coverage achievable, such as attack containment or recovery (UAV replacement and/or role swap) speed. When an attack occurs during a mission, our model can be used to evaluate

4. For example the DJI Zenmuse H20T thermal camera has $40.6^{\circ}$ DFOV [21], and Flir VUE TZ20 has $95^{\circ}$ FOV [22].

5. The EU limit for operation in the 'open' category is $120 \mathrm{~m}$ [23]. the extent to which the mission can be expected to succeed despite the attack.

5.5.1. Pre-planning. When planning a mission, the SWN component of our overall model enables answering questions such as: What is the impact of an attack if no containment approach is used, vs with containment? Answering this is achieved by comparing the performance of two SWN versions, with and without containment. How many replacement vehicles are required to ensure recovery to a specific number of vehicles after an attack? This can be answered by running the analysis varying the number of replacement UAVs. Can varying the formation of the vehicles/partitioning the network reduce the chances of a disruption due to an attack? Such analysis would involve varying the SWN input parameters to reflect different assumptions on vehicle connectivity and formations, and comparing the results across the different setups.

The overall mission analyses, conducted with our full modeling methodology, also require consideration for mission-level parameters outside of the SWN model, such as the maximum mission duration, UAV specifications, and the time of the attack occurrence. The full model enables the estimation of mission success metrics and consequently the evaluation of mission-level issues such as: What is the overall coverage reachable after an attack, and how does it change with the maximum mission length or the time of attack? How does the loss of a UAV to compromise affect the mission coverage if the vehicle in question was acting in a sensor role, or in a relay role?

Although we have focused here on the availability of UAVs over time, the metrics obtainable with the SWN modeling are not limited to this. Other metrics that can be readily obtained from the SWN analysis include the likelihood of system states of interest, such as the loss of $\mathrm{N}$ sensor vehicles to compromise, or the likelihood that at least the minimum number of relays are online to ensure mission information is passed to the MC. Such metrics could be used to answer questions such as: How many replacement UAVs are required to keep the probability of mission failure due to an attack below a certain threshold? Additionally, in future work we plan to explore multiple and/or repeat attacks in the model. This would enable evaluating the impact of repeat attacks on the number of redundant replacement vehicles required and the time it takes to recover the mission. From the mission performance perspective it would allow estimating the number of attacks that can be sustained before the mission can no longer be fulfilled.

5.5.2. During attack analysis. During an attack, the focus is on the likelihood of mission success and degree of mission completion. Our modeling can help answer questions such as: Can the mission continue, or should it be aborted? Answering this question is achieved by estimating the expected mission coverage given the attack using the SWN model, and comparing the result to the mission requirement. This also involves considering the remaining mission time, as part of the overall mission coverage estimation. We assume such analysis will be performed by the mission command based on data received from the vehicles. What share of the coverage requirement can be achieved given the attack? The current progress 
TABLE 2. AREA COVERAGE OVER TIME FOR AN INDIVIDUAL VEHICLE, HA/MIN

\begin{tabular}{|c|c|c|c|c|c|c|c|c|c|c|c|c|c|c|c|c|}
\hline \multirow{2}{*}{$\begin{array}{c}\text { Speed }(\mathrm{m} / \mathrm{s}) \\
\text { AOV }\end{array}$} & \multicolumn{4}{|c|}{5} & \multicolumn{4}{|c|}{10} & \multicolumn{4}{|c|}{15} & \multicolumn{4}{|c|}{20} \\
\hline & $40^{\circ}$ & $80^{\circ}$ & $95^{\circ}$ & $150^{\circ}$ & $40^{\circ}$ & $80^{\circ}$ & $95^{\circ}$ & $150^{\circ}$ & $40^{\circ}$ & $80^{\circ}$ & $95^{\circ}$ & $150^{\circ}$ & $40^{\circ}$ & $80^{\circ}$ & $95^{\circ}$ & $150^{\circ}$ \\
\hline Altitude & & & & & & & & & & & & & & & & \\
\hline $50 \mathrm{~m}$ & 1.1 & 2.5 & 3.3 & 11.2 & 2.2 & 5.0 & 6.5 & 22.4 & 3.3 & 7.6 & 9.8 & 33.6 & 4.4 & 10.1 & 13.1 & 44.8 \\
\hline $100 \mathrm{~m}$ & 2.2 & 5.0 & 6.5 & 22.4 & 4.4 & 10.1 & 13.1 & 44.8 & 6.6 & 15.1 & 19.6 & 67.2 & 8.7 & 20.1 & 26.2 & 89.6 \\
\hline $120 \mathrm{~m}$ & 2.6 & 6.0 & 7.9 & 26.9 & 5.2 & 12.1 & 15.7 & 53.7 & 7.9 & 18.1 & 23.6 & 80.6 & 10.5 & 24.2 & 31.4 & 107.5 \\
\hline
\end{tabular}

towards the goal and estimate of the coverage reachable in the remaining time can be a useful metric for mission planners and operators, especially if the mission goal is flexible. This is a direct output of our mission analysis.

\section{Analysis results}

This section shows the results of the analysis for our case study. We describe them in two parts. First, we introduce the attack impact evaluation using the Petri net model, which shows the expected impact of an attack on the vehicles participating in the mission, in a time window starting from the attack occurrence. This is explained on its own, without reference to the other components of our mission viability evaluation method. Second, we show results for the overall mission analysis, and how the results are affected by parameters relating to the mission.

\subsection{Analysis using the SWN model}

We report here the results of the impact analysis for sensor vehicles only (i.e. without discussing relays), as they are the basis for overall mission coverage, and because the insights obtained from the results for relay vehicles are qualitatively similar. The analysis in the next section combines both the sensor and relay vehicles into the mission coverage rate. Table 3 shows the parameter values for our SWN model used in the analysis of the mission considered in our case study.

Fig. 4 shows how the attack impacts the number of vehicles taking part in the mission over time, excluding those that have been compromised by the attack. The figure depicts the expected number of sensor vehicles, i.e. tokens representing sensor vehicles in the place $U A V s$ of the Petri net, evaluated over the model time window using the transient solution provided by the GreatSPN solver [20]. The various panels of the figure show the effect on the expected number of sensor vehicles from varying different parameters values. When a parameter is not varied, it is kept at its baseline level; the baseline parameter values used are: del_cont=20; del_prpg=2; del_rec $=599$; del_swp=40; Red=3.

We can observe in Fig. 4 that:

- Containment delay has a large impact on the effectiveness of the defense: early on there is a difference of around three sensor vehicles between the slower and faster containment cases (Fig. 4a). This effect can persist until the end of the evaluation time if there are not enough replacement UAVs to replace all the compromised ones.

- A longer propagation delay reduces the impact on sensor vehicle numbers, as it increases the likelihood that containment occurs before propagation (Fig. 4b).

- Replacement delay causes some difference in the short run, with longer delays decreasing the number of sensors in use, but this closes out toward the end of the evaluation time frame (Fig. 4d).

- Variations in the swap delay have a minor impact on the expected number of sensor UAVs available in our case study (Fig. 4e).

- The number of replacement vehicles has a huge impact if containment is slow. With a large number of replacement vehicles, the impact of an attack is eventually mitigated even if it propagates to the whole network, while a system with faster containment can manage with fewer replacements (Fig. 4h).

\subsection{Mission success analysis}

Using the coverage rate function (1) to convert the SWN analysis results to UAV numbers, Fig. 5 shows the coverage impacts from attacks. The patterns are similar to those in the sensor numbers plots, but with somewhat deeper dips due to the impact compromised relays have on the coverage rate.

Table 4 shows the total expected coverage reachable during a mission when sustaining a cyber attack, using the approach explained in Section 5.4. The table illustrates the impacts from individual vehicle's assumed coverage rate (as in Table 2), maximum mission time, and the time of the attack. The table is calculated for the sample mission setup (as in Fig. 1a-1b and Fig. 2) with two interconnected relays and eight sensor vehicles, four of which are in the video role, and the baseline SWN parameter values. The same SWN evaluation of attacks is applied to all cases. Table 4 shows that a) the same attack occurring earlier in the mission time significantly limits the overall expected coverage achieved relative to a later attack if the number of replacement vehicles is low $(\operatorname{Red}=3)$, but a high redundancy reduces this effect $(\operatorname{Red}=9)$; b) The total area that can be covered within a mission time varies greatly with the individual UAV coverage rate.

\section{Conclusion}

We have introduced an approach for evaluating the impacts of cyber attacks on the viability of a mission involving the deployment of multiple UAVs that can communicate with each other. Our method provides a structured approach for mission planning support and viability evaluation, helping to determine: a) how many vehicles are needed for a mission with a given coverage requirement, and how this varies with parameters such as mission connectivity configuration, maximum mission time, vehicle specifications, and the occurrence time of a possible attack; b) how many redundant vehicles should be available to act as replacements in case of an attack; c) how different parameters impact the total coverage reachable within a mission time, which enables a better 


\begin{tabular}{|c|c|c|}
\hline $\begin{array}{l}\text { del } l_{\text {cont }}: \text { containment delay, del } l_{\text {cont }} \in\{4,20\},\left(\text { del }_{\text {cont }}=1 / r_{\text {cont }}\right) \\
\text { del } l_{\text {prpg }}: \text { propagation delay, del } \text { prpg }_{\text {prpo }} \in\{2,5\},\left(\text { del }_{\text {prpg }}=1 / r_{\text {prpg }}\right) \\
\text { del } l_{\text {rec }}: \text { replacement delay, del } l_{\text {rec }} \in\{300,599,901\},\left(\text { del }_{\text {rec }}=1 / r_{\text {rec }}\right) \\
\text { del } l_{\text {swp }}: \text { swap delay, del } l_{\text {swp }} \in\{40,150,300\},\left(\text { del }_{\text {swp }}=1 / r_{\text {swp }}\right) \\
\text { del } l_{\text {desp }}: \text { despatch delay, del } l_{\text {desp }}=25,\left(\text { del }_{\text {desp }}=1 / r_{\text {desp }}\right)\end{array}$ & $\begin{array}{l}\text { Red: replacement (redundant) UAVs, Red } \in\{0,3,6,9\} \\
X_{i}: \text { excess/redundancy in role }\left(1 \text { over limit), } X_{i}=L_{i}+1\right. \\
\quad t: \text { model time, seconds, } t \in[0,1200] \\
r_{i}: \text { UAV with role } i, r_{1}: \text { relay, } r_{2}: \text { sensor (temp/video) } \\
E_{i}: \text { entry UAV role: } E_{1}=0, E_{2}=1\end{array}$ & $\begin{aligned} & N_{i}: N_{1}=2, N_{2}=8 \\
& L_{i}: L_{1}=2, L_{2}=4 \\
& S_{s i}: S_{11}=1, S_{12}=0 \\
& \quad: S_{21}=1, S_{22}=3 \\
& \quad: S_{31}=0, S_{32}=4\end{aligned}$ \\
\hline
\end{tabular}

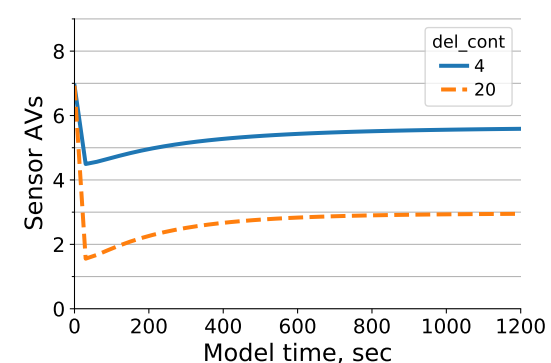

(a) Containment delay $\{4,20\}$

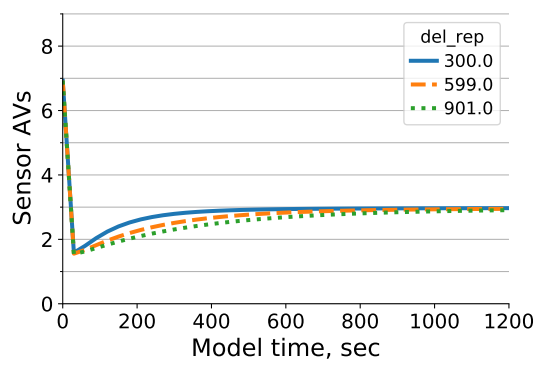

(d) Replacement delay $\{300,599,901\}$

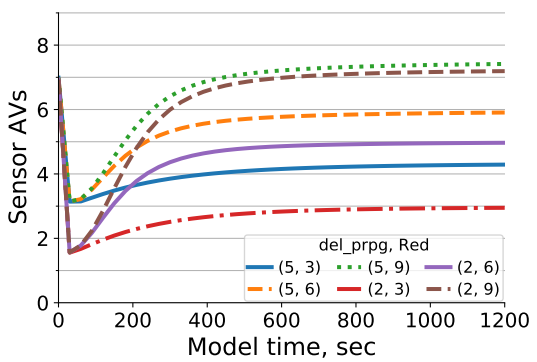

(g) Propagation delay and replacement vehicles

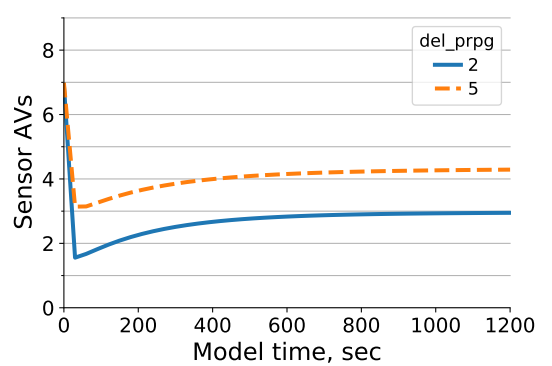

(b) Propagation delay $\{2,5\}$

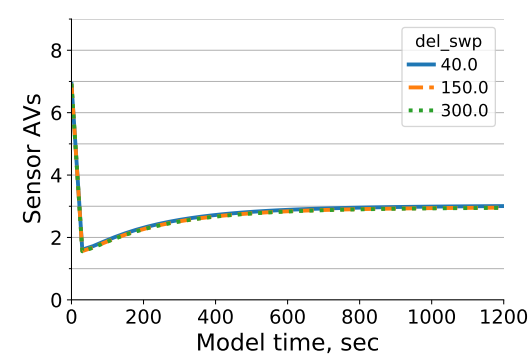

(e) Swap delay $\{40,150,300\}$

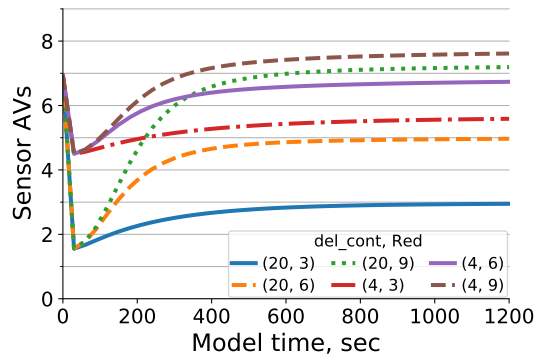

cles

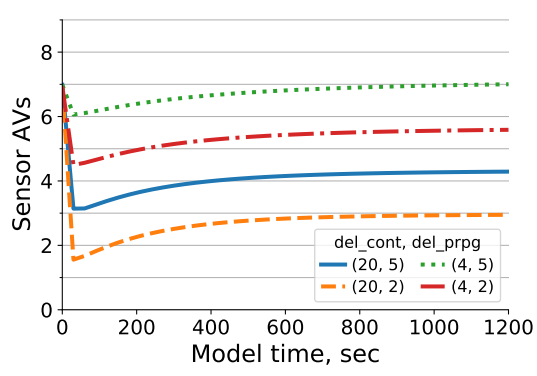

(c) Containment and propagation delays

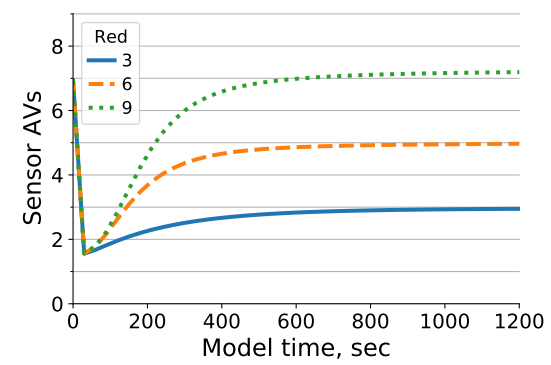

(f) Count of replacement vehicles $\{3,6,9\}$

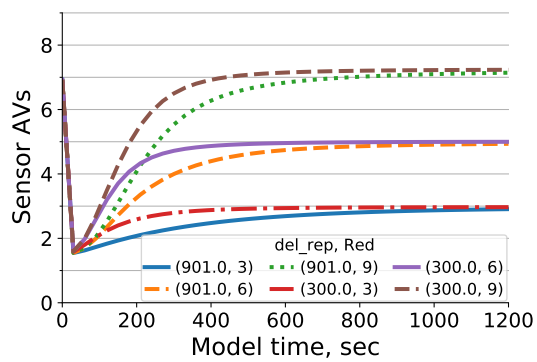

(i) Replacement delay and replacement vehicles

Figure 4. Expected number of sensor vehicles online. Baseline parameter values: del_cont=20; del_prpg=2; del_rec=599; del_swp=40; Red=3

understanding of the impact of an attack and can help in prioritizing different capabilities.

We have shown the use of this method in a firefighting case study scenario informed by actual data on fires and UAV capabilities. The results highlight the importance of the speed of containment and appropriate capacity for recovery in the form of redundant vehicles. While their importance as the key determinants of overall mission performance during attacks is to be expected, our method provides a way to quantify their impact and to estimate their relative importance where a trade-off is possible, as faster containment can make up for fewer redundant vehicles and vice versa.

Although we have shown a specific implementation, our method can be applied more generally. Most importantly, the approach to modeling attack progression and impact analysis with Petri nets is applicable to other settings that involve several components that are similar in how they function and how an attack can laterally proceed across them. This applies e.g. in multi-UAV deployments, or self organizing robotic swarms. We also demonstrated how the Petri net model can be used in conjunction with other parts of our methodology to conduct analysis of success at the level of the mission, via the use of a performance metric relating to mission progress. We believe that the estimated coverage metric we used can be applied to other distributed (aerial) missions where area coverage is important, such as search and rescue or disaster assessment [1], with only small adjustments. For other domains a different metric might be required, but the base approach would still apply.

In future work we plan to extend our analysis to study the impact of several successive attacks, whether resulting from the same vulnerability or different ones. This could leverage another useful feature of Petri nets, enabling compact modeling of repeat attacks. 


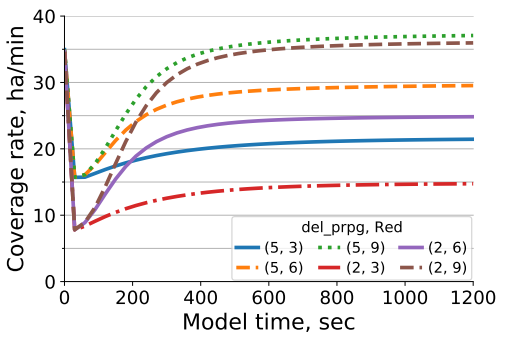

(a) Propagation delay and replacement vehicles

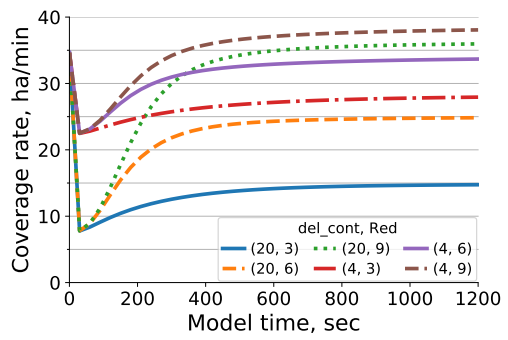

(b) Containment delay and replacement vehicles

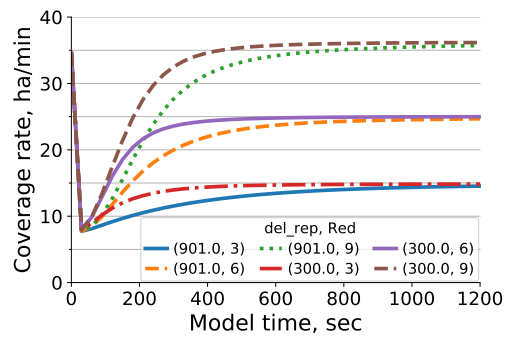

(c) Replacement delay and replacement vehicles

Figure 5. Coverage rates during attack. Baseline parameter values: del_cont=20; del_prpg=2; del_rec=599; del_swp=40; Red=3

TABLE 4. TOTAL EXPECTED MISSION COVERAGE, HA

\begin{tabular}{|c|c|c|c|c|c|c|c|c|c|c|c|c|c|}
\hline \multirow{2}{*}{\multicolumn{2}{|c|}{$\begin{array}{c}\text { UAV coverage, ha/min } \\
\text { Max time, min }\end{array}$}} & \multicolumn{3}{|c|}{1} & \multicolumn{3}{|c|}{5} & \multicolumn{3}{|c|}{10} & \multicolumn{3}{|c|}{15} \\
\hline & & 20 & 30 & 40 & 20 & 30 & 40 & 20 & 30 & 40 & 20 & 30 & 40 \\
\hline Replacements & $\begin{array}{c}\text { Attack time, min } \\
5\end{array}$ & 41.1 & 54.6 & 69.7 & 205.7 & 273.2 & 348.4 & 411.4 & 546.5 & 696.7 & 617.1 & 819.7 & 1045.1 \\
\hline \multirow[t]{2}{*}{3} & 15 & 66.7 & 81.1 & 94.6 & 333.4 & 405.7 & 473.2 & 666.8 & 811.4 & 946.5 & 1000.2 & 1217.1 & 1419.7 \\
\hline & 25 & 80.0 & 106.7 & 121.1 & 400.0 & 533.4 & 605.7 & 800.0 & 1066.8 & 1211.4 & 1200.0 & 1600.2 & 1817.1 \\
\hline \multirow{3}{*}{9} & 5 & 67.4 & 99.6 & 135.4 & 337.2 & 498.1 & 677.1 & 674.3 & 996.2 & 1354.1 & 1011.5 & 1494.3 & 2031.2 \\
\hline & 15 & 72.7 & 107.4 & 139.6 & 363.4 & 537.2 & 698.1 & 726.8 & 1074.3 & 1396.2 & 1090.3 & 1611.5 & 2094.3 \\
\hline & 25 & 80.0 & 112.7 & 147.4 & 400.0 & 563.4 & 737.2 & 800.0 & 1126.8 & 1474.3 & 1200.0 & 1690.3 & 2211.5 \\
\hline
\end{tabular}

\section{References}

[1] H. Shakhatreh, A. H. Sawalmeh, A. Al-Fuqaha, Z. Dou, E. Almaita, I. Khalil, N. S. Othman, A. Khreishah, and M. Guizani, "Unmanned Aerial Vehicles (UAVs): A Survey on Civil Applications and Key Research Challenges," IEEE Access, vol. 7, pp. $48572-$ 48 634, 2019.

[2] J. San-Miguel-Ayanz, T. Durrant, R. Boca, P. Maianti, G. Libertá, T. Artés Vivancos, D. Jacome Felix Oom, A. Branco, D. De Rigo, D. Ferrari, H. Pfeiffer, R. Grecchi, D. Nuijten, and T. Leray, Forest Fires in Europe, Middle East and North Africa 2019. Luxembourg, 2020, JRC122115: EUR 30402 EN, Publications Office of the European Union, 2020.

[3] B. Nassi, R. Bitton, R. Masuoka, A. Shabtai, and Y. Elovici, "SoK Security and Privacy in the Age of Commercial Drones," in Proc. 2021 IEEE Symp. Security and Privacy (SP), 2021, pp. 73-90.

[4] J.-P. Yaacoub and O. Salman, "Security analysis of drones systems: Attacks, limitations, and recommendations," Internet of Things, $\mathrm{p}$. 100218, 2020.

[5] K. L. Best, J. Schmid, S. Tierney, J. Awan, N. M. Beyene, M. A. Holliday, R. Khan, and K. Lee, "How to Analyze the Cyber Threat from Drones," RAND Corporation, Santa Monica, CA, United States, Tech. Rep., 2020.

[6] M. Albanese and S. Jajodia, "A Graphical Model to Assess the Impact of Multi-Step Attacks," Journal of Defense Modeling and Simulation: Applications, Methodology, Technology, vol. 15, no. 1, pp. 1-15, 2017.

[7] J. Soikkeli, L. Muñoz González, and E. Lupu, "Efficient Attack Countermeasure Selection Accounting for Recovery and Action Costs," in Proceedings of the 14th International Conference on Availability, Reliability and Security, ser. ARES '19. ACM, 2019.

[8] B. B. Madan and K. S. Trivedi, "Security modeling and quantification of intrusion tolerant systems using attack-response graph," Journal of High Speed Networks, vol. 13, no. 4, pp. 297-308, 2004.

[9] B. Li, R. Lu, K.-K. R. Choo, W. Wang, and S. Luo, "On Reliability Analysis of Smart Grids under Topology Attacks: A Stochastic Petri Net Approach," ACM Trans. Cyber-Phys. Syst., vol. 3, no. 1, Aug. 2018.

[10] O. M. Dahl and S. D. Wolthusen, "Modeling and Execution of Complex Attack Scenarios Using Interval Timed Colored Petri Nets," in Proc. 4th IEEE International Workshop on Information Assurance, IWIA 2006, 2006, pp. 157-168.
[11] D. H. Collins and A. V. Huzurbazar, "Petri net models of adversarial scenarios in safety and security," Military Operations Research, vol. 24, no. 3, pp. 27-48, 2019.

[12] A. Y. Javaid, W. Sun, V. K. Devabhaktuni, and M. Alam, "Cyber security threat analysis and modeling of an unmanned aerial vehicle system," 2012 IEEE International Conference on Technologies for Homeland Security, HST 2012, pp. 585-590, 2012.

[13] I. Linkov and A. Kott, "Fundamental concepts of cyber resilience: Introduction and overview," in Cyber Resilience of Systems and Networks. Springer, 2019, pp. 1-25.

[14] M. Bruneau, S. E. Chang, R. T. Eguchi, G. C. Lee, T. D. O'Rourke, A. M. Reinhorn, M. Shinozuka, K. Tierney, W. A. Wallace, and D. Von Winterfeldt, "A framework to quantitatively assess and enhance the seismic resilience of communities," Earthquake spectra, vol. 19, no. 4, pp. 733-752, 2003.

[15] A. A. Ganin, E. Massaro, A. Gutfraind, N. Steen, J. M. Keisler, A. Kott, R. Mangoubi, and I. Linkov, "Operational resilience: Concepts, design and analysis," Scientific Reports, vol. 6, pp. 1-12, 2016.

[16] European Union. (2000) European Forest Fire Information System (EFFIS). Accessed: 2021-04-27. [Online]. Available: https://effis.jrc.ec.europa.eu/applications/data-and-services

[17] A. Mas-Colell, M. D. Whinston, and J. R. Green, Microeconomic Theory. New York: Oxford University Press, 1995.

[18] M. A. Marsan, G. Balbo, G. Conte, S. Donatelli, and G. Franceschinis, Modelling With Generalised Stochastic Petri Nets. Chichester: J. Wiley \& Sons Ltd., 1995.

[19] G. Chiola, C. Dutheillet, G. Franceschinis, and S. Haddad, "Stochastic Well-Formed Colored Nets and Symmetric Modeling Applications," IEEE Transactions on Computers, vol. 42, no. 11, pp. 1343-1360, 1993.

[20] E. G. Amparore, G. Balbo, M. Beccuti, S. Donatelli, and G. Franceschinis, "30 Years of GreatSPN," Principles of Performance and Reliability Modeling and Evaluation, pp. 227-254, 2016.

[21] DJI. (2020) DJI Zenmuse H20T specs. [Online]. Available: https://www.dji.com/uk/zenmuse-h20-series/specs

[22] Flir. (2020) Flir VUE TZ20 specs. [Online]. Available: https: //www.flir.co.uk/products/vue-tz20/

[23] Commission Delegated Regulation (EU) 2019/945 of 12 March 2019 on unmanned aircraft systems and on third-country operators of unmanned aircraft systems, C/2019/1821. 